\title{
Detection of Halogenated Flame Retardants in Polyurethane Foam by Particle Induced X-ray Emission
}

Adam M. Maley ${ }^{a}$, Kyle A. Falk ${ }^{\mathrm{a}}$, Luke Hoover ${ }^{\mathrm{a}}$, Elly B. Earlywine ${ }^{\mathrm{a}}$, Michael D. Seymour ${ }^{\mathrm{e}}$, Paul A. DeYoung ${ }^{b}$, Arlene Blum ${ }^{c}$, Heather M. Stapleton ${ }^{d}$ and Graham F. Peaslee ${ }^{a \S}$

${ }^{\text {a }}$ Department of Chemistry, Hope College, 35 E. $12^{\text {th }}$ Street, Holland, MI 49423, USA

${ }^{\mathrm{b}}$ Department of Physics, Hope College, 27 Graves Place, Holland, MI 49423, USA

${ }^{c}$ Green Science Policy Institute, Box 5455, Berkeley, CA, 94705, USA

${ }^{\mathrm{d}}$ Nicholas School of the Environment, Duke University, LSRC Box 90328, Durham, NC, 27708, USA

${ }^{\S}$ Corresponding Author

peaslee@ hope.edu, +1-616-395-7117

\begin{abstract}
A novel application of Particle-Induced X-ray Emission (PIXE) has been developed to detect the presence of chlorinated and brominated flame retardant chemicals in polyurethane foams.

Traditional Gas Chromatography-Mass Spectrometry (GC-MS) methods for the detection and identification of halogenated flame retardants in foams require extensive sample preparation and data acquisition time. The elemental analysis of the halogens in polyurethane foam performed by PIXE offers the opportunity to identify the presence of halogenated flame retardants in a fraction of the time and sample preparation cost. Through comparative GC-MS and PIXE analysis of 215 foam samples, excellent agreement between the two methods was obtained. These results suggest that PIXE could be an ideal rapid screening method for the presence of chlorinated and brominated flame retardants in polyurethane foams.
\end{abstract}

Key Words: Flame retardants, PIXE, GC-MS, polyurethane foam 


\section{Introduction}

In recent years, there have been a large number of publications that highlight the potential hazards of halogenated flame retardants (FRs) in the environment ${ }^{1-5}$ and the health concerns from human exposure. ${ }^{6-13}$ Several of these commercial products have been voluntarily removed from the US market, including polybrominated biphenyls (PBBs, 1973), ${ }^{14}$ pentabrominated diphenyl ethers and octabrominated diphenyl ethers (Penta-BDEs and Octa-BDEs, 2004) ${ }^{15}$ and decabrominated diphenyl ether (Deca-BDE, 2013). ${ }^{16}$ Some chlorinated FRs, such as Tris (1,3dichloro-2-propyl) phosphate (TDCPP), have been identified as suspected human carcinogens, ${ }^{17-}$ 18 and products containing foam treated with TDCPP must be labeled as such under California's Proposition 65. ${ }^{19}$ Many of the current replacement FRs entering the US market are halogenated compounds with similar chemistry, including decabromodiphenyl ethane (DBDPE), hexabromocyclododecane (HBCD), bis(2,4,6,-tribromphenoxy)ethane (BTBPE), 2-ethylhexyl 2,3,4,5-tetrabromobenzoate (TBB), (2-ethylhexyl) tetrabromophthalate (TBPH), Tris (2chloroethyl) phosphate (TCEP), and Tris (1-chloro-2-propyl) phosphate (TCPP). ${ }^{20-23}$ All of these chemicals, both those that are new and those being phased out, belong to a class of chemicals known as organohalogens and many, with the exception of the phosphates, are defined as persistent organic pollutants. ${ }^{24}$ Several of these are stable in the environment, bioaccumulate, and have been shown to be potentially toxic and/or carcinogenic in experiments with animals. ${ }^{25-}$ 30

While halogenated FR use in polyurethane foams is prohibited in many countries, the US and UK have adopted furniture fire-safety standards that are so stringent that these laws cannot be easily met without the use of halogenated FR chemicals. For example, one of the major reasons for the wide-spread use of FR chemicals in the United States is the California Home 
Furnishings Bureau's flammability standard, Technical Bulletin 117 (TB 117). This 1975

legislation requires that a piece of polyurethane foam in upholstered furniture withstand an open flame for 12 seconds. ${ }^{31}$ However, since so many recent scientific studies have shown these FR chemicals can be toxic and bioaccumulate in the human body, and because this story has begun to hit the mass media, ${ }^{32-34}$ the state of California has changed its legislation to adopt a smolder standard which does not require the use of FRs in products containing polyurethane foam. This revised standard, TB 117-2013, also exempts specific juvenile foam products, such as car seats, highchairs, changing pads, and infant mattresses, to help protect those at highest risk from these chemicals. $^{35}$

This rapidly-changing political landscape in fire-safety legislature and increasing public awareness about the potential toxicity and prevalence of these FR chemicals in our environment has led to an increasing demand to test polyurethane foam products for the presence of halogenated FR chemicals. The traditional analytical method used to identify FR chemicals in foam is Gas Chromatography-Mass Spectrometry (GC-MS). For this chemical test, a polyurethane foam sample is typically immersed in an organic solvent to extract the FR chemicals, and then the extract is reduced in volume by evaporation techniques, and injected into a GC-MS column for identification. Comparison with standard solutions can then yield quantitative evaluations and precise identification of which chemicals are present in the polyurethane foams. ${ }^{36-38}$ The halogenated FR chemicals normally require extended analysis time in the GC-MS method because the FRs tend to pass slowly through most GC columns. The solvent extraction, evaporation, and comparison with standard solutions combined with approximately 20-minute column analysis time means that the practical use of GC-MS to identify the presence of FR chemicals in foams can be tedious and expensive. 
The goal of this project was to develop a rapid method to screen for the presence of chlorinated and brominated FRs using PIXE. While one can test for the presence of brominated FR chemicals with hand-held X-ray Fluorescence (XRF) instruments because the elemental bromine X-rays have sufficient energy to be measured in air, these measurements are not as reliable for chlorinated FRs due to chlorine's relatively low-energy X-rays. ${ }^{39}$ The development of an in vacuo ion beam analysis method allows the concentrations of both chlorine and bromine to be quantitatively measured in a polyurethane foam matrix, and the sample preparation and analysis time can be reduced from hours per sample to minutes with a comparable reduction in cost. While PIXE analysis alone cannot identify the specific FR used, there are no typical sources of halogens within polyurethane foams that contribute to chlorine and bromine levels greater than 2-5\% (by weight). This means that for rapid screening of large numbers of foam samples, such as those requested by concerned consumers of foam-filled furnishings, clothing, bedding, and automobiles, PIXE analysis could provide a viable method to test for the presence of these chemicals of concern.

There are several other potential screening methods that could identify both $\mathrm{Br}$ and $\mathrm{Cl}$ in polyurethane foams, including in-vacuum XRF systems which should be as sensitive as PIXE. However, these in-vacuum XRF systems are no longer portable and therefore the analysis method is roughly equivalent to PIXE. Newer, more advanced tabletop XRF systems are capable of measuring $\mathrm{Cl}$ in air, but preliminary results in a follow-up study to this work indicates that it is less reliable quantitatively than in vacuo studies for $\mathrm{Cl}$ measurements. ${ }^{40}$ Better methods that not only perform elemental identification but also rapidly identify the FR chemicals might include techniques such as Surface Ionization with Time-of-Flight Mass Spectrometry in vacuum, or Desorption Electrospray Ionization in air. However, the primary purpose for this 
study is to provide a method for existing Ion Beam Analysis facilities to provide an important environmental monitoring capability while, currently, there are only limited rapid assay options.

\section{Materials and Methods}

\subsection{Materials}

A TDCPP (95\% pure) quantification standard was purchased from TCI America (Portland, OR). Penta-BDE, TBB, and TCPP standards (99\% pure) were purchased from AccuStandard (New Haven, CT). TCEP (97\% pure) and Triphenyl phosphate (TPP, 99\% pure) standards were purchased from Sigma-Aldrich (St. Louis, MO). All solvents used in this study were purchased from Sigma-Aldrich and were HPLC grade.

Polyurethane foam standards with known concentrations $(8.15 \%$ and $4.76 \%$, by mass) of TDCPP were provided by ICL Industrial Products (Beer Sheva, Israel).

\subsection{Sample Collection}

Polyurethane foams samples, ranging from approximately 1 to $10 \mathrm{~cm}^{3}$ in volume, were cut from couch cushions, chairs, pillows, mattress padding, and other pieces of furniture that were donated by the public. The foam samples were wrapped in aluminum foil, stored in a zippered plastic bag, labeled, and mailed to Hope College for analysis. A majority of the samples were collected by students working with the Green Science Policy Institute and the University of California, Berkeley in Berkeley, CA and the remaining samples were collected by Hope College students. A total of 215 polyurethane foam samples of known provenance were analyzed by both GC-MS and PIXE. To help confirm our findings, a subset of 24 foams were also analyzed by GC-MS at Duke University as part of a previously published study. ${ }^{36}$ These were "samples of 
opportunity" that had already been completely analyzed by a reputable research group, for which there existed replicate samples in order to augment our study.

\subsection{GC-MS Methods}

\subsubsection{Sample Extractions}

FR chemicals were extracted from the polyurethane foam samples by soaking a $\sim 10 \mathrm{mg}$ piece of foam in $10 \mathrm{~mL}$ of dichloromethane (DCM) and sonicating for 15 minutes. The DCM solution was then filtered into a clean vial and reduced in volume by rotary evaporation. DCM extractions were first run in the GC-MS at a 100X dilution. If no FR chemicals were detected at this concentration, further dilutions at $10 \mathrm{X}, 5 \mathrm{X}$, and $1 \mathrm{X}$ were run until FR chemicals could be detected in the sample or the sample was run at full concentration.

\subsubsection{Sample Analysis}

Samples were analyzed using an Agilent gas chromatograph (model 6890) mass spectrometer (model 5973). Foam sample extracts were analyzed using electron impact mode (GC/ EI-MS) over a scan range of 45-800 amu for the detection of TCEP, TCPP, TDCPP, TPP, TBB, and Penta-BDE. All EI spectra were compared to the NIST Mass Spectral Library. External calibration standards were used for quantification. A $0.25 \mathrm{~mm}$ (i.d.) X $30 \mathrm{~m} \mathrm{HP}-5 \mathrm{~ms}$ capillary column coated with 5\% phenyl methyl siloxane (Agilent model 19091s-433, $0.25 \mu \mathrm{m}$ film thickness) was used for separation of the analytes. The oven temperature was set to initial temperature of $100^{\circ} \mathrm{C}$ with a temperature ramp of $20^{\circ} \mathrm{C} /$ min to a temperature of $200{ }^{\circ} \mathrm{C}$, followed by a temperature ramp of $8^{\circ} \mathrm{C} / \mathrm{min}$ to a final temperature of $300{ }^{\circ} \mathrm{C}$. Table 1 shows the retention times in the GC for the various FRs measured in this study. 


$\begin{array}{cc}\text { FR } & \text { Approximate } \\ \text { RCEP } & 7.2 \\ \text { TCPP } & 7.7 \\ \text { TDCPP } & 13.4 \\ \text { TPP } & 14.1,15.4 \\ \text { Penta-BDE } & 17.3 \\ \text { TBB } & 17.9\end{array}$

Table 1. Retention times of FR chemicals in the GC column.

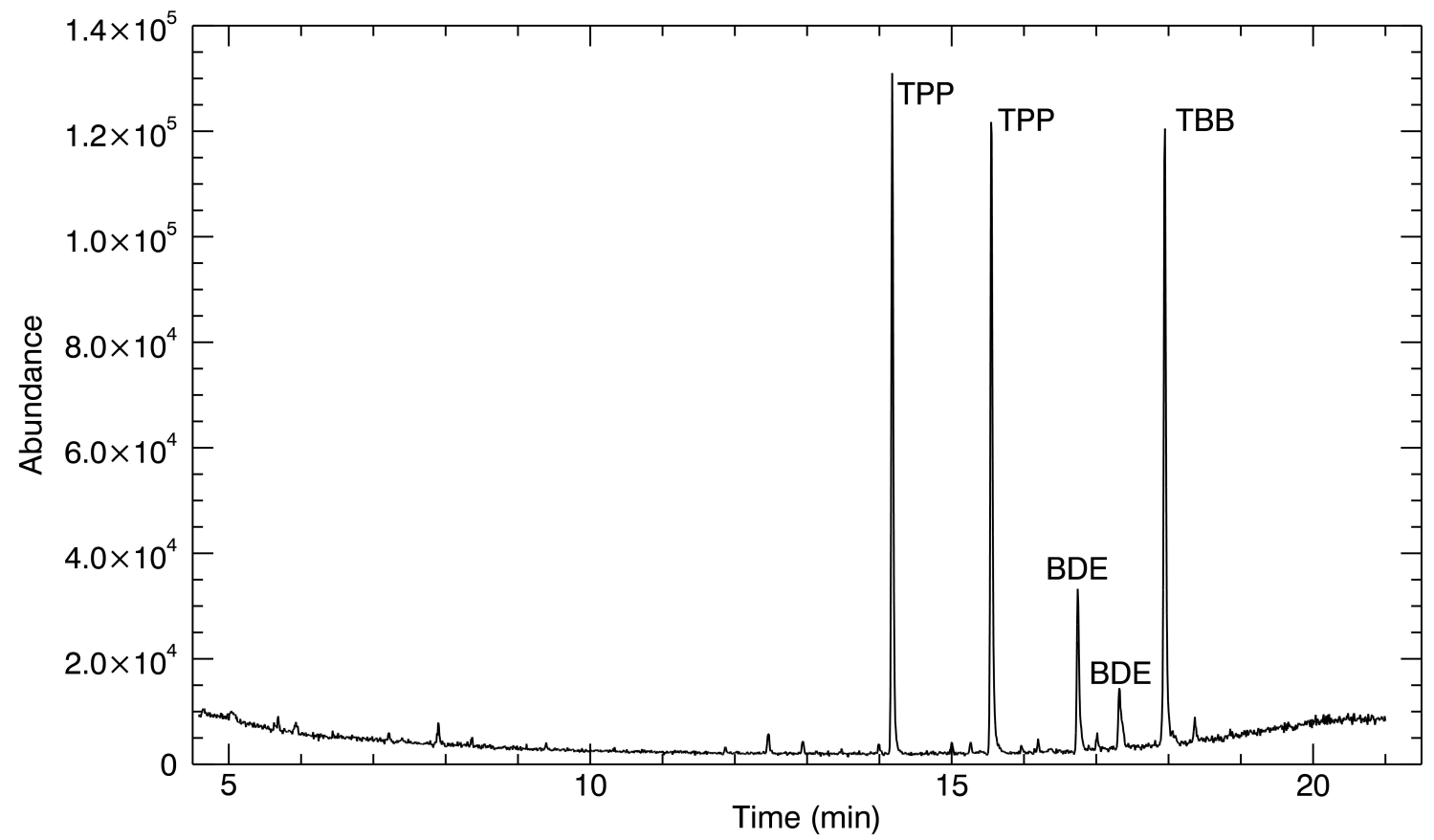

Figure 1. GC chromatogram of a typical sample containing TPP, Penta-BDEs and TBB. PIXE analysis of this sample indicates brominated FRs.

\subsection{PIXE Methods}

\subsubsection{Sample Preparation}

Small pieces of foam, approximately $50 \mathrm{~mm}^{3}$ in volume, were cut out from each larger foam sample and were manually adhered to an aluminum target ladder with double-sided 
adhesive tabs. Over a dozen samples could be mounted per target ladder, together with a sample cut from the ICL foam of known TDCPP concentration. It was not necessary to homogenize or otherwise manipulate the foam samples.

\subsubsection{PIXE Analysis}

Using the Hope College Ion Beam Analysis Laboratory (5SDH Pelletron Accelerator, National Electrostatics Corp, Middleton, WI), each foam sample was irradiated with approximately $1-5 \mathrm{nA}$ of $3.4 \mathrm{MeV}$ protons for $60 \mathrm{sec}$ in a high-vacuum scattering chamber. Characteristic elemental X-rays emitted from each sample were detected by a Si(Li) detector (Ortec, model SLP-10180-ST) located at $135^{\circ}$ with respect to the beam axis. There was a 0.002 " Mylar filter placed between the target and the X-ray detector to suppress low-energy X-rays. The effective detection threshold allowed one to record silicon $\mathrm{K}_{\alpha} \mathrm{X}$-rays, but only semi-quantitative concentrations of each element lighter than sulfur could be obtained. Chlorine and bromine $\mathrm{K}_{\alpha}$ X-rays were quantified for each foam sample using the commercial peak-fitting program GUPIXWin ${ }^{\circledR} .^{41}$ Examples of PIXE spectra from a foam sample containing both chlorinated and brominated FRs and from a FR-free foam, for comparison, are shown in Figure 2. In the example shown in Figure 2, the target foams were mounted on a ladder with a hole in it for the beam to pass through. When mounted on a solid aluminum target latter, iron, copper, and chromium peaks (which are known contaminants in the aluminum target ladders) appear, which indicate that the foam samples are not thick enough to stop the proton beam entirely. Since the X-ray energies of these contaminants are so easily resolved separately from the halogen peaks, there was no need to switch to a non-metallic target ladder. 


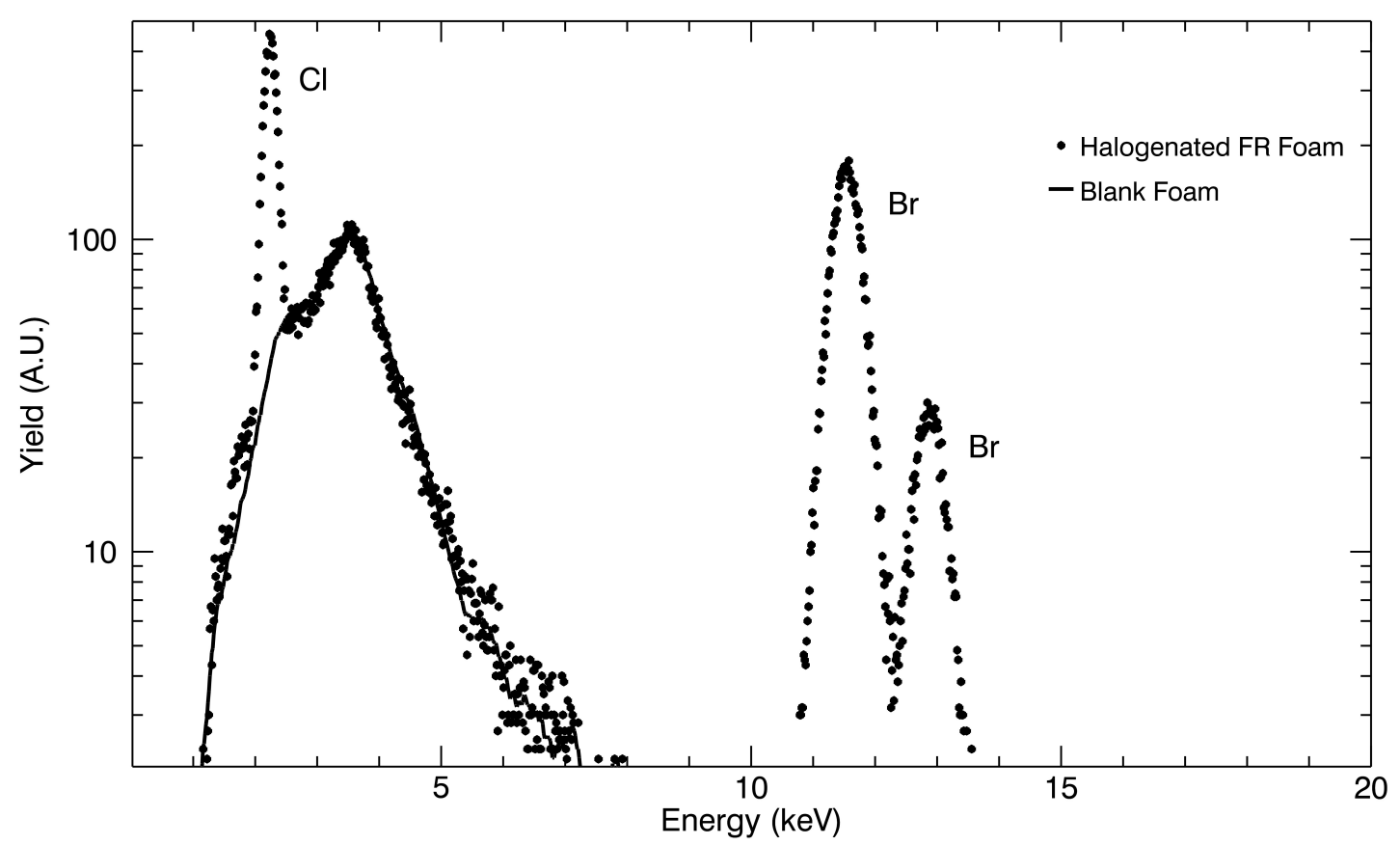

Figure 2. Typical PIXE spectra of polyurethane foams containing chlorine and bromine peaks due to $\sim 4 \%$ by mass FR (dotted trace) and a foam with no FR additive (solid trace). Note the yaxis is semi-logarithmic to demonstrate the low-background found for both chlorine and bromine.

\subsection{Standards}

In order to obtain quantitative results, foam samples with known concentrations of flame retardants were used to standardize results with GUPIXWin ${ }^{\circledR}$. Only calibrated standards were available for TDCPP in foam, so the bromine concentrations were estimated using the relative $\mathrm{X}$ ray production cross sections and X-ray detection efficiencies provided by GUPIXWin ${ }^{\circledR}$. Since the foam standards have a very similar organic matrix to the unknown samples, the standards are used to account for varying beam conditions and to normalize the quantitative yields of chlorine and bromine X-rays to the incident beam intensity for each sample. Faraday cup measurements of the beam intensity are made before and after each set of 12 foam samples on a target ladder, 
and quantification of chlorine and bromine detected within each sample is estimated to be on the order of $\pm 10 \%$ by weight using this beam intensity measurement technique. This has been confirmed by replicate measurements of the standards.

\section{Results \& Discussion}

The foam standards were used to determine a practical detection limit for the presence or absence of halogenated FRs in foam samples. As seen in Figure 2, both chlorine and bromine have X-ray intensities much greater than the X-ray counts from metals contaminants in the aluminum target ladders (iron, copper, and chromium) indicating the presence of a halogenated FR in the sample. Occasionally some metals were seen in various polyurethane foams, such as trace levels of iron, or intentional addition of antimony and zinc as inorganic FRs. Through this analysis of the foam standards, a chlorine concentration $\geq 0.3 \%$ by weight was used to indicate the presence of a chlorinated FR and a bromine concentration $\geq 0.1 \%$ by weight was used to indicate the presence of a brominated FR. These levels were chosen to unambiguously distinguish all measured samples with flame retardants intentionally added to the foam, and the slightly higher value used for chlorine reflects the higher Bremsstrahlung background visible in all foam samples peaked at around $3.5 \mathrm{keV}$ for this particular detector geometry and filter thickness.

Replicate PIXE analyses of the two foam standards for chlorine are shown in Table 2.

These results indicate that there is a precision of about $6-8 \%$ on these polyurethane targets, which is typical of ion beam surface analysis measurements. This uncertainty is typically attributed to uncertainty in the absolute beam intensity delivered to target from measurement to measurement. 


\begin{tabular}{|c|c|c|}
\cline { 2 - 3 } \multicolumn{1}{c|}{} & $\begin{array}{c}\text { TDCPP - } \\
\text { Standard 1 }\end{array}$ & $\begin{array}{c}\text { TDCPP - } \\
\text { Standard 2 }\end{array}$ \\
\hline Nominal $(\% \mathrm{Cl})$ & 8.15 & 4.76 \\
\hline $\mathrm{N}$ & 26 & 25 \\
\hline PIXE Avg. $(\% \mathrm{Cl})$ & 8.052 & 4.670 \\
\hline St. Dev. $(\% \mathrm{Cl})$ & 0.523 & 0.364 \\
\hline RSD $(\% \mathrm{Cl})$ & 6.5 & 7.8 \\
\hline
\end{tabular}

Table 2. Results for replicate PIXE analyses on two polyurethane foam standards. The nominal results reported from the manufacturer compare well with the absolute PIXE measurements.

Of the 215 post-consumer foam samples that were collected and analyzed by both PIXE and GC-MS, $65 \%$ of the samples contained chlorinated or brominated FRs according to the PIXE analysis and 64\% contained chlorinated or brominated FRs according to the GC-MS analysis. In other words, in the 131 samples where a FR was found to be present by GC-MS, PIXE was also able to correctly identify these samples as containing FRs. However, there were 6 foam samples where PIXE obtained a false positive test and 4 foam samples where PIXE obtained a false negative test, assuming the GC-MS analysis to be entirely correct. The summary of results is shown in Table 3.

\begin{tabular}{|c|c|c|c|c|}
\cline { 2 - 5 } \multicolumn{1}{c|}{} & \multicolumn{3}{c|}{ FR Identification by GC-MS Detection } \\
\hline $\begin{array}{c}\text { FR Identification by } \\
\text { Elemental PIXE Detection }\end{array}$ & Cl FR Only & Br FR Only & Cl and Br FR & None \\
\hline Cl Only & $\mathbf{6 5}$ & 0 & 0 & 4 \\
\hline Br Only & 0 & $\mathbf{4 4}$ & 4 & 2 \\
\hline Cl and Br & 7 & 6 & $\mathbf{8}$ & 0 \\
\hline None & 3 & 0 & 1 & $\mathbf{7 1}$ \\
\hline
\end{tabular}

Table 3. Comparison of results for 215 samples analyzed by both PIXE and GC-MS. FR presence determined by PIXE is listed in horizontal rows and FR presence determined by GCMS is listed in vertical columns. Each table entry, which indicates number of samples, matches each possible PIXE result with each possible GC-MS result. Numbers along the diagonal (bolded) indicate perfect agreement between PIXE and GC-MS. 
There are several possible causes for the small (6/215) false positive rate observed, but the most likely reason is the post-consumer addition of salts to the foam samples. These samples came from used mattress pads and couch cushions, where bodily fluids might have added inorganic salt to the sample. There is also potential for the deliberate addition of inorganic salts for other purposes during the manufacturing of the foam, or the cross-contamination of inorganic salts used as FR chemicals in the textile coverings of the foam samples, which may be treated with FR chemicals as well. Also, in foam samples that were at one time covered by a FR-treated fabric (particularly the $\mathrm{Br}$ false positive samples, because of the widespread use of decaBDE in textiles), there is the possibility that some of the covering FR chemicals could transfer to the surface of the polyurethane foam, and since PIXE is a surface-analysis technique, it may register a signal which is not present throughout the bulk volume of foam digested for GC-MS analysis. The most likely explanation for the small (4/215) false negative rate observed is the inhomogeneous distribution of FR chemicals within a small piece of foam. PIXE analysis is a surface technique, and if only one side of the sample were to contain a halogenated FR chemical from a textile cross contamination, for example, PIXE would register the presence of a halogen on the surface, while the solvent extraction on the larger bulk samples would yield only trace amounts of halogenated FR chemical from the same sample. Sample processing or labeling errors are also a possibility in this preliminary test for both the GC-MS and the PIXE analyses.

Since the samples used in GC-MS analysis are solvent-extracted from replicate pieces of foam, a more representative concentration of FR throughout the entire foam may be possible. Yet, PIXE analysis of replicate foam samples has indicated that the concentration of FRs can vary by considerably more than $20 \%$, depending on what portion or side of the foam is analyzed 
with beam. This difference in FR concentrations throughout a piece of foam could also lead to false positive and negative detections of FRs with PIXE.

It was also observed that 21 samples ( $\sim 15 \%$ of the FR-treated foams) contained distinct X-ray signatures from both chlorine and bromine, while the GC-MS analysis found only 8 of these samples to contain two different halogenated FR chemicals. These results are also included in Table 3. This is not an error in the identification of FR chemicals, but either a limit of detection issue for the GC-MS method of identifying a second low-concentration halogenated chemical in the presence of a much more prevalent primary FR chemical, or the presence of inorganic halogens or species that are not amenable to GC-MS analysis. It could also represent a reagent contamination issue in the original production of these FR chemicals. In the PIXE analysis, the ratio of chlorine to bromine or bromine to chlorine for these samples is usually between 10:1 and 2:1, which means only a fraction of the secondary halogen is present compared to the primary halogen present. While it is clear the contamination occurs at the manufacturing level, either the GC-MS background is too high to observe the secondary compound or FR chemicals are not entirely pure of the secondary halogen.

The identification of the types of FRs found in the 215 foam samples by GC-MS are shown in Table 4. This distribution of FR chemicals is consistent with the types of FR chemicals found in other recent studies of foam products in the $\mathrm{US}^{37}$.

\begin{tabular}{|c|c|c|}
\hline $\begin{array}{c}\text { FR detection } \\
\text { in GC-MS }\end{array}$ & Number of Samples & $\%$ of Samples \\
\hline TCEP & 17 & $8 \%$ \\
\hline TCPP & 22 & $10 \%$ \\
\hline TDCPP & 61 & $28 \%$ \\
\hline TPP & 62 & $28 \%$ \\
\hline Penta-BDE & 36 & $16 \%$ \\
\hline TBB & 23 & $10 \%$ \\
\hline
\end{tabular}


Table 4. Various FRs identified in the 215 polyurethane foam samples. There are more than the 138 samples listed in Table 3, because some foams had multiple FRs within a single sample.

\section{Conclusions}

An efficient ion beam analysis method for screening polyurethane foam samples for the presence of chlorinated and brominated FRs has been developed. While analysis by GC-MS will remain the industry standard for identification of which specific halogenated FR chemical is present, the ability to analyze hundreds of foam samples per day for the presence of FR chemicals in foam at any PIXE facility allows the analysis costs per sample to be reduced dramatically. The sample preparation takes typically less than one minute per sample, and the Xray acquisition can be done in one or two minutes per sample. There are larger capital costs compared to GC-MS analysis, but for existing facilities, this possibility puts the realm of rapid product screening within reach for most consumers of products that contain polyurethane foams. It is likely that the demand for such product testing will only increase from consumers, retailers, environmental organizations, and even manufacturers wanting to test their supply chain as awareness of the environmental toxicity of these FR chemicals becomes known.

PIXE is also a non-destructive analytical technique so it is possible to use it as a prescreening method for determining presence of a chlorinated or brominated FRs. This would cut down on analysis times of large sample sets since only some samples would be subjected to a complete GC-MS analysis. One limitation to this study is the ability of PIXE to detect nonhalogenated flame retardants, which are becoming more prevalent in the market (e.g. TPP). ${ }^{37}$ Although it was not done in this study because replicate samples were used for each analytical technique, the foam could be sequentially analyzed first by PIXE and subsequently by GC-MS (a destructive analysis) if absolute identification of the FRs present in a foam sample is necessary. 
Although these are semi-volatile organic compounds embedded in the polyurethane foam manufacturing process, and though they do out-gas modestly under high-vacuum proton irradiation, there are always abundant quantities of FR chemical compounds left in the foam samples for subsequent chemical analysis if needed.

Future work will expand this technique to other types of FR-treated materials, survey more samples to build a larger comparative GC-MS database of results, and incorporate other analytical techniques to try to uniquely identify the FR chemicals present within each sample.

\section{Acknowledgements}

The authors would like to acknowledge ICL Industrial Products for donating foam samples with known concentrations of TDCPP. Funding provided by the National Science Foundation (NSF-RUI \#0969058 and \#1306074, and NSF CAREER CHE-0952768), the US Environmental Protection Agency (GL00E01116-0) and the Department of Energy (DESC0007352).

\section{References}

1. Hale, RC; La Guardia, MJ; Harvey, E; Gaylor, MO; Mainor, TM. Brominated flame retardant concentrations and trends in abiotic media. Chemosphere 2006, 64 (2), 181-186.

2. Law, RJ; Allchin, CR; de Boer, J; Covaci, A; Herzke, D; Lepom, P; Morris, S;

Tronczynski, J; De Wit, CA. Levels and trends of brominated flame retardants in the European environment. Chemosphere 2006, 64 (2), 187-208.

3. de Wit, CA; Alaee, M; Muir DCG. Levels and trends of brominated flame retardants in the Artic. Chemosphere 2006, 64 (2), 209-233.

4. Shaw, SD; Berger, ML; Brenner, D; Kannan, K; Lohmann, N; Papke, O. Bioaccumulation of polybrominated diphenyl ethers and hexabromocyclododecane in the northwest Atlantic marine food web. Sci. Total. Environ. 2009, 407 (10), 3323-3329.

5. Meng, XZ; Blasius, ME; Gossett, RW; Maruya, KA. Polybrominated diphenyl ethers in pinnipeds stranded along the southern California coast. Environ. Pollut. 2009, 157 (10), 2731-2736. 
6. Blum A; Ames, BN. Flame-retardant additives as possible cancer hazards. Science 1977, 195 (17), 17-23.

7. Sjodin, A; Patterson, DG; Bergman, A. A review on human exposure to brominated flame retardants-particularly polybrominated diphenyl ethers. Environ. Int. 2003, 29 (6), 829-839.

8. Hites, RA. Polybrominated diphenyl ethers in the environment and in people: A metaanalysis of concentrations. Environ. Sci. Technol. 2004, 38 (4), 945-956.

9. Inoue, K; Harada, K; Takenaka, K; Uehara, S; Kono, M; Shimizu, T; Takasuga, T; Senthilkumar, K; Yamashita, F; Koizumi, A. Levels and concentration ratios of polychlorinated biphenyls and polybrominated diphenyl ethers in serum and breast milk in Japanese mothers. Environ. Health. Persp. 2006, 114 (8), 1179-1185.

10. Fangstrom, B; Athanassiadis, L; Odsjo, T; Noren, K; Bergman, A. Temporal trends of polybromianted diphenyl ethers and hexabromocyclododecane in milk from Stockholm mothers, 1980-2004. Mol. Nutr. Food Res. 2008, 52 (2), 187-193.

11. Petreas, M; Nelson, D; Brown, FR; Goldberg, D; Hurley, S; Reynolds, P. High concentrations of polybrominated diphenylehters (PBDEs_ in breast adipose tissue of California women. Environ. Int. 2011, 37 (1), 190-197.

12. Gascon, M; Fort, M; Martinez, D; Carsin, AE; Forns, J; Grimalt, JO; Marina, LS; Lertxundi, N; Sunyer, J; Vrijheid, M. Polybrominated Diphenyl Ethers (PBDEs) in breast milk and neuropsychological development in infants. Environ. Health Persp. 2012, 120 (12), 1760-1765.

13. Buttke, DE; Wolkin, A; Stapleton, HM; Miranda, ML. Associations between serum levels of polybrominated diphenyl ether (PBDE) flame retardants and environmental and behavioral factors in pregnant women. J. Expo. Sci. Env. Epid. 2013, 23 (2), 176-182.

14. United States Environmental Protection Agency. Technical Fact Sheet- Polybrominated diphenyl ethers (PBDEs) and polybrominated biphenyls (PBBs). EPA 505-F-11-007. Washington, DC, 2012.

15. Tullo, A. Great Lakes to phase out flame retardants. Chem. Eng. News 2003, 81 (45), 13.

16. EPA. DecaBDE phase-out initiative. http://www.epa.gov/opptintr/existingchemicals/pubs/actionplans/deccadbe.html Accessed: May 16, 2013.

17. World Health Organization. EHC 209: Flame retardants: Tris-(Chloropropyl)Phosphate and Tris-(2-Chloroethyl)phosphate. 1998.

18. van der Veen, I; de Boer, J. Phosphorus flame retardants: Properties, production, environmental occurrence, toxicity, and analysis. Chemosphere 2012, 88 (10), 11191153.

19. Proposition 65. http://oehha.ca.gov/prop65.html Accessed: May 28, 2013.

20. United States Environmental Protection Agency. Furniture flame retardancy partnership: Alternatives for low-density polyurethane foam. EPA 742-R-05-002B. Washington, DC, 2005.

21. Stapleton, HM; Allen, JG; Kelly, SM; Konstantinov, A; Klosterhaus, S; Watkins, D; McClean, MD; Webster, T. Alternate and new brominated flame retardants detected in U.S. house dust. Environ. Sci. Technol. 2008, 42 (18), 6910-6916.

22. Stapleton, HM; Klosterhaus, S; Eagle, S; Fuh, J; Meeker, JD; Blum, A; Webster, TF. Detection of organophosphate flame retardants in furniture foam and U.S. house dust. Environ. Sci. Technol. 2009, 43 (19), 7490-7495. 
23. Allen, JG; Stapleton, HM; Vallarino, J; McNeely, E; McClean, MD; Harrad, SJ; Rauert, $\mathrm{CB}$; Spengler, JD. Exposure to flame retardant chemicals on commercial airplanes. Environ. Health 2013, 12 (17).

24. Ritter, L; Solomon, KR; Forget, J; Stemeroff, M; O’Leary, C. Persistent organic pollutants. The International Programme on Chemical Safety. http://www.chem.unep.ch/pops/ritter/en/ritteren.pdf Accessed: May 16, 2013.

25. Zhou, T; Ross, DG; DeVito, MJ; Crofton, KM. Effects of short-term in vivo exposure to polybrominated diphenyl ethers on thyroid hormones and hepatic enzyme activities in Weanling rats. Toxicol. Sci. 2001, 61 (1), 76-82.

26. Birnbaum, LS; Staskal, DF. Brominated flame retardants: Cause for concern? Environ. Health. Persp. 2004, 112 (1), 9-17.

27. Viberg, H; Fredriksson, A; Eriksson, P. Neonatal exposure to the brominated flameretardant, 2,2',4,4',5-pentabromodiphenyl ether, decreases cholinergic nicotinic receptors in hippocampus and affects spontaneous behaviour in the adult mouse. Enviorn. Toxicol. Phar. 2004, 17 (2), 61-65.

28. Costa, LF; Fiordano, G. Developmental neurotoxicity of polybrominated diphenyl ether (PBDE) flame retardants. Neurotoxicology 2007, 28 (6), 1047-1067.

29. He, P; Wang, AG; Xia, T; Gao, P; Niu, Q; Guo, LJ; Xu, BY; Chen, XM. Mechanism of the neurotoxic effect of PBDE-47 and interaction of PBDE-47 and PCB153 in enhancing toxicity in SH-SY5Y cells. Neurotoxicology 2009, 30 (1), 10-15.

30. He, P; Wang, AG; Xia, T; Gao, P; Niu, Q; Guo, LJ; Chen, XM. Mechanisms underlying the developmental neurotoxic effect of PBDE-147 and the enhanced toxicity associated with its combination with PCB153 in rats. Neurotoxicology 2009, 30 (6), 1088-1095.

31. Technical Bulletin 117. http://www.bhfti.ca.gov/industry/117.pdf Accessed: May 16, 2013.

32. Callahan, P; Roe, S; Hawthorne, M. Tribute watchdog: Playing with fire. Chicago Tribune http://www.media.apps.chicagotribune.com/flames/index.html Accessed: May 16, 2013.

33. Slater, D. How dangerous is your couch? The New York Times http://www.nytimes.com/2012/09/09/magazine/Arlene-blums-crusade-against-householdtoxins.html Accessed May 16, 2013.

34. Gross, L. Flame retardants in consumer products are linked to health and cognitive problems. The Washington Post http://www.articles.washingtonpost.com/2013-0415/national/38550740_1_flame-retardants-pdbes-treated-products Accessed: May 16, 2013.

35. Technical Bulletin 117-2013, Initial Statement of Reasons. http://www.bhfti.ca.gov/about/laws/isr.pdf Accessed: May 16, 2013.

36. Stapleton, HM; Kosterhaus, S; Keller, A; Ferguson, PL; van Bergen, S; Cooper, E; Webster, TF; Blum, A. Identification of flame retardants in polyurethane foam collected from baby products. Environ. Sci. Technol. 2011, 45 (12), 5323-5331.

37. Stapleton, HM; Sharma, S; Getzinger, G; Ferguson, PL; Gabriel, M; Webster, TF; Blum, A. Novel and high volume use flame retardants in US couches reflective of the 2005 PentaBDE phase out. Environ. Sci. Technol. 2012, 46 (24), 13432-13439.

38. Fang, M; Webster, TF; Gooden, D; Cooper, EM; McClean, MD; Carignan, C; Makey, C; Stapleton, HM. Investigating a novel flame retardant known as V6: Measurements in baby products, house dust, and car dust. Environ. Sci. Technol. 2013, 47 (9), 4449-4454. 
39. Allen, JG; McClean, MD; Stapleton, HM; Webster, TF. Linking PBDEs in house dust to consumer products using x-ray fluorescence. Environ. Sci. Technol. 2008, 42 (11), 42224228.

40. Savagian, LR; O’Connell, H.; Greve, E; Liao, Y; Maley, AM; Gearhart J; Peña, K; Peaslee, GF; Identification of Halogenated Flame Retardants in Automotive Foam Seats (in preparation 2015)

41. Campbell, JL; Boyd, NI; Grassi, N; Bonnick, P; Maxwell, JA. The Guelph PIXE software package IV. Nucl. Instrum. Meth. B 2010, 268 (20), 3356-3363. 